\title{
El origen del aprendizaje a lo largo de la \\ vida como medida de inclusión. \\ Los casos de Alemania y España
}

\author{
Sianes-Bautista, Alicia \\ Universidad Nacional de Educación a Distancia, Sevilla, España \\ asianes2@alumno.uned.es
}

\section{Resumen}

En la actualidad nos hallamos inmersos en una sociedad compleja y cada vez más heterogénea dominada por el dinamismo y el devenir. Sin embargo, al mismo tiempo también se está luchando en pro de lograr una homogeneización de los sistemas educativos en Europa. En este marco contextual se atisban situaciones de desigualdad de oportunidades que confluye en la necesidad de instaurar medidas inclusivas destinadas a minimizar estas d esigualdades. El aprendizaje a lo largo de la vida ya es hoy en día un concepto ampliamente extendido en el mundo occidental que atiende a la importancia de seguir construyendo el aprendizaje de forma paulatina a lo largo de todo el periodo vital. Tratando de evitar de esta manera que el proceso formativo de los individuos cese una vez terminados los estudios obligatorios o tras haber alcanzado una edad determinada. En la presente aportación recurrimos a la metodología comparada para abordar el origen del aprendizaje a lo largo de la vida en dos países con historia y contextos dispares, como es el caso de España y Alemania. El objetivo principal reside en poder presentar las diferencias y similitudes del aprendizaje a lo largo de la vida en estos países desde su surgimiento, pues es el momento en el que por vez primera se contempla como una necesidad. Entre los resultados obtenidos destaca la inestabilidad política en ambos países objeto de estudio a inicios del siglo XIX, el analfabetismo de gran parte de la población y la necesidad de mano de obra cualificada para la inserción profesional. A modo de colofón destacamos el fuerte vínculo entre la educación a lo largo de la vida y la educación de adultos, pues fue una medida de inclusión social que se dirigió principalmente las personas en edad adulta.

\section{Abstract}

Nowadays we are immersed in a complex society which is increasingly being more heterogeneous and it is dominated by dynamism and development. However, at the same time, efforts are also being made to achieve homogenization of the education systems in Europe. In this contextual framework, situations of inequality of opportunities are seen, which converge in the need to establish inclusive measures aimed at minimizing these inequalities. Lifelong learning is nowadays in the western world a widespread concept that addresses the importance of gradually continuing the learning process throughout the life cycle. Trying to avoid this way that the training process of the individuals ceases once completed the compulsory studies or after having reached a certain age. In this contribution, we resort to the comparative methodology to address the origin of lifelong learning in two countries with different histories and contexts, such as Spain and Germany. The main objective is to present the differences and similarities of lifelong learning in these countries since their emergence, because it is the moment when it has been seen as a necessity for the first time. The main obtained results show the political instability in both studied countries at the beginning of the 19th century, the illiteracy of a substantial part of the population and stand out the need for skilled workforce for professional insertion. As a conclusion, we emphasize the strong link between lifelong learning and adult education, because it was a measure of social inclusion which was mainly addressed to people in adulthood.

Palabras clave: Aprendizaje a lo largo de la vida, Alemania, España, inclusión.

Keywords: Lifelong learning, Germany, Spain, inclusion. 


\section{INTRODUCCIÓN}

El Aprendizaje a lo largo de la Vida, en adelante ALV, es un concepto muy utilizado en el discurso anglosajón que conforma un paradigma complejo en conceptualización y terminología. Son múltiples los términos empleados, en discursos políticos y debates académicos, como educación continua, educación de adultos, educación para todos, educación permanente, formación a lo largo de la vida, etc. (Torres, 2003, citado en García Ruíz, 2005). El origen del ALV se remonta a finales de la I Guerra Mundial, extendiéndose su influencia durante la segunda década del S. XX, principalmente en aspectos ligados a la promoción política, al debate sobre la extensión de los derechos humanos a las mujeres y a colectivos de clase obrera (Field, 2000).

El objetivo del ALV consiste en «garantizar el futuro económico (...), contribuir a la civilización de la sociedad y desarrollar la dimensión espiritual de la vida, y promover la ciudadanía activa» (DfEE, 1998:7). García Ruíz (2005) indica la democratización del saber a todos como uno de los atributos más valiosos e intrínsecos del ALV. Sin embargo, todavía hay quienes consideran que la educación de adultos es «un ámbito débil y generalmente menospreciado de la escena educativa oficial» (Giere y Piet, 1997:3-4, citado por Field, 2000:22 y García Ruíz, 2005)

El concepto de Educación Permanente se encuentra dividido entre los que lo conciben como un Educación a lo largo de la Vida y los que lo admiten como ALV (Etcheverría Arroyo, 2007).

Siguiendo sus aportaciones, en el primer caso se percibe una influencia directa del Informe de la Comisión Internacional de Educación para el Siglo XXI (Informe Delors) en el que se expresa un continuum educativo descrito como:

«...las funciones asignadas a la educación y las múltiples formas que puede tomar, englobando, desde la infancia al fin de la vida, todas las ofertas que permiten a cada persona acceder a un conocimiento dinámico del mundo, de los demás y de sí mismo» interrelacionando los considerados cuatro «....pilares fundamentales del aprendizaje: aprender a conocer, a hacer, a vivir y a convivir con los demás y aprender a ser» (UNESCO, 1995).

Mientras que en el segundo caso, se palpa la relevancia que le fue conferida por la OCDE al ALV, comprendiendo el desarrollo personal y social en todas sus formas y contextos.

«Consiste en una aproximación sistémica que acompaña los niveles de conocimientos y de competencias a adquirir, independientemente de la edad de los sujetos. Su énfasis radica en la necesidad de motivar a la infancia para instruirse durante toda la vida y orientar los esfuerzos de manera que todos los adultos tengan la oportunidad de hacerlo» (OCDE, 1996).

El Informe Faure Aprender a Ser (1972), fue un hito en relación con el establecimiento y la declaración oficial de los principios de la ELV. La educación debía producirse durante toda la vida y no ceñirse a un periodo concreto y dirigido hacia minorías. Los ideales humanistas de la ELV presentes en el Informe Faure fueron sustituidos por la promoción de fuerza laboral conducente a la productividad y la eficiencia para incrementar la competitividad económica y el nivel de vida (García Ruíz, 2005).

Field (2000:134) planteaba cuatro estrategias esenciales para implantar el ALV en los sistemas educativos: reformular el rol de la escolaridad, incrementar la participación en el aprendizaje adulto, construir una ciudadanía activa invirtiendo en capital social y la búsqueda de significado.

Para alcanzar el objetivo esencial de esta contribución, el cual reside en presentar las diferencias y similitudes del ALV en Alemania y en España, se ha optado por el empleo del método comparado, propio de la Educación Comparada. Aunque es una disciplina heterogénea en cuanto a objeto y enfoques teóricos y metodológicos, hemos seguido las fases metodológicas propuestas por Llorent Bedmar (2002), a partir de los planteamientos de García Garrido (1996).

\section{APRENDIZAJE A LO LARGO DE LA VIDA EN ALEMANIA}

A principios del siglo XIX Alemania poseía un fuerte foco de influencia educativa prusiana que irradiaba en el resto de países germanófonos centroeuropeos. En 1811 tiene lugar la paradigmática reforma humboldtiana, trayendo consigo la supresión de las escuelas elementales y estableciéndose 
un sistema escolar graduado único para todos (Spranger, 1960). La escuela desempeña adecuadamente su función alfabetizadora, y a partir del siglo XX empieza a preocuparse por la extensión cultural y la asistencia en pro de la inserción laboral. Antes de la II Guerra Mundial, se distinguían tres fases en el ámbito del ALV.

En la primera, artesanos y campesinos se ven amenazados por la Revolución Industrial, surgiendo asociaciones con el objetivo de conservar su producción. Sin embargo, esta situación cambia, principalmente debido al incremento del proletariado industrial a mediados del siglo XIX. Entonces, las políticas formativas abarcaban la educación elemental y la formación profesional como vía para superar dificultades económicas y laborales. Adicionalmente cobra importancia la formación política para implantar un orden social democrático en las estructuras feudales del momento (Mollenhauer, 1973). Esta etapa finaliza con la Revolución de marzo de 1848.

La segunda fase abarca la Restauración y el Imperio Alemán, hasta 1918. Tras la Revolución de 1848 incrementan las diferencias entre burgueses y proletarios, aunque en la educación de adultos se conservan actitudes colaborativas para lograr la constitución democrática del Estado, contra la Restauración. En 1869 se disuelven los congresos comunes, suponiendo la separación entre la Educación Popular de los liberal-burgueses y de los socialistas y socialdemócratas. En 1871 se funda la Sociedad para la extensión de la Educación Popular, como consecuencia de los «cada vez más agudos perfiles de las clases sociales» (Gómez R. de Castro, 1994:182), generando que la educación popular burguesa abandone los principios antifeudalistas, anticlericales y emancipatorios. Paralelamente a la formación burguesa, se desarrolla una educación para obreros adultos, facilitadora de la inserción social e influyente para el cambio hacia una sociedad socialista. Un ejemplo sería la Formación de funcionarios en los sindicatos, sancionada por la ley de 1920 (Feidel-Mertz, 1975).

A partir de 1918 la tercera fase, durante la República de Weimar, la Sociedad para la extensión de la educación popular realza la formación individual y humanista. En las escuelas superiores populares cada vez son más los asistentes oyentes ocasionales, además de asociaciones de trabajadores, incrementándose su inclusión. Se buscaba el perfeccionamiento y el desarrollo de las cualidades personales. En 1919 la Constitución de Weimar creó la Volkhochschule desde el Gobierno Federal, los Länder y ayuntamientos. Esta es una institución que está muy extendida y actualmente sigue siendo la institución dedicada al ALV más representativa de Alemania, contando con 899 centros (Volkhochschule, 2018). Su objetivo es «la adquisición de conocimientos y la profundización en las experiencias vividas, la transmisión de información contrastada, la potenciación del pensamiento propio e independiente de las personas y el ejercicio de las capacidades creativas» (Gómez R. de Castro, 1994:183).

En el ALV en Alemania se distinguen dos periodos: el primero hasta mediados del siglo XX, cuando las nuevas iniciativas, concepciones e intentos por retomar el rumbo de la formación de adultos ya existente en la República de Weimar brillan por su ausencia. Asimismo, la carencia de fundamentos legales y ayudas presupuestarias agravaba la situación, por lo que esta educación se contempla como un lujo que no debe promocionarse por el sistema de formación general de la República Federal. Tras la II Guerra Mundial, a consecuencia de las problemáticas económicas, de la Segunda Revolución Industrial y de la necesidad de mano de obra cualificada se volcaron esfuerzos enfocados a la formación profesional industrial, pasando de esta forma la formación política a un segundo plano (Gómez R. de Castro, 1994).

Actualmente (Eurydice, 2018) el ALV es una cuestión que cada vez cobra mayor importancia en Alemania debido en gran parte al desarrollo demográfico. En relación con esto, la formación profesional continua institucionalizada dirige el posterior desarrollo de las cualificaciones individuales, y las reorientaciones en relación con las mismas. Asimismo, se prevé que el desarrollo, el reconocimiento y la certificación de competencias serán cada vez más importantes en el futuro, al igual que los nuevos aprendizajes. De igual modo, la educación continua abarca también los dominios generales, vocacionales y sociopolíticos. Especialmente las interacciones en relación con el desarrollo y la transferencia de las competencias en el ALV, también aumentan. Para dar respuesta a la amplia gama de demandas de la educación continua, se ha desarrollado una estructura diferenciada. En este sentido, las instituciones de educación continua y de adultos ofertan una amplia variedad de cursos y formación en la educación académica general, vocacional, política y continua. 


\section{APRENDIZAJE A LO LARGO DE LA VIDA EN ESPAÑA}

A inicios del siglo XIX comienza en España el sistema escolar estatal, una mejora que, aunque importante, era ya tardía pues incluso actualmente existen personas que han llegado a la edad adulta sin haber sido alfabetizadas (Gómez R. de Castro, 1994). Como consecuencia de esto, la primera aproximación a la educación de adultos surge de la necesidad de extender la educación primaria a personas que no tuvieron la oportunidad de acceder al sistema escolar en su infancia. A partir de entonces se establecen tres directrices que inspiraron la educación de adultos y el ALV hasta la LOGSE para coadyuvar a su inclusión (Gómez R. de Castro, 1994:168). La primera era recuperar las deficiencias en la formación general, la segunda facilitar la inserción profesional y la tercera posibilitar la participación en la vida social y política.

La primera vez que se hace alusión a esta educación en España es en 1821 en el reglamento de Instrucción pública. No obstante, fue la ley de 15 de julio de 1838 la primera que hace alusión a las escuelas de adultos. El currículum solía ceñirse a lectura, escritura y cálculo, pretendiendo cubrir la ausencia de alfabetización de este alumnado. Esta cuestión se acentúa posteriormente mediante la Ley Moyano (1857), con la cual se precisa que las autoridades provinciales y municipales colaboren en el fomento, la financiación y la responsabilidad de las escuelas para adultos (Gómez R. de Castro, 1994).

Durante la Restauración (1874-1903), se diferencian tres fases. En la primera (1874-1879) se adapta la legislación educativa a la constitución de 1876; la segunda (1879-1893) se concibe como la fase dialéctica más activa, caracterizada por la progresiva imposición de ideas liberales; finalmente, tras la tragedia de 1898, liberales y conservadores cooperan en pro de condensar los principios formulados en su política legislativa, culminando con la Reforma de Romanones.

En 1906 se incluye en el Real Decreto de 4 de octubre, una campaña contra el analfabetismo extendiéndose al colectivo adulto, aunque apenas se aplicó. A modo de subsanación se promulga el decreto de 19 de mayo de 1911(Gómez R. de Castro, 1994). En la segunda mitad del siglo XX, persiste el interés estatal por la regulación legislativa de esta educación, aunque la II República supuso que las normativas reguladoras vigentes hasta entonces se frenaran, guiándose por el regeneracionismo y apoyándose en el prestigio de la Institución Libre de Enseñanza. Debido a la elevada desescolarización y al 32,4\% de analfabetismo (Gómez R. de Castro, 1994:173), este aprendizaje pasa a un segundo plano.

Durante el franquismo destaca la eficaz lucha contra el analfabetismo y, además, la Educación de Adultos queda enmarcada en la estrategia de Educación Permanente, donde la alfabetización se da por superada y pasa a centrarse en la formación cultural. A partir de los 60, la UNESCO y otros organismos internacionales influyen notablemente pues, en la Orden Ministerial de 1953 se dispone el establecimiento de clases de adultos mixtas servidas por maestros y maestras de las escuelas nacionales; el establecimiento de escuelas volantes en núcleos de población diseminada; y la organización de colonias y campamentos para combatir el analfabetismo en sectores específicos (Gómez R. de Castro, 1994:175). El Boletín Oficial del Estado publica el 5 de septiembre de 1963 el Decreto de Presidencia, mediante el que se pone en marcha una campaña a nivel estatal, provincial y local dirigida por el Ministerio de Educación basada en el derecho de todo hombre a la Educación Permanente, la igualdad de oportunidades para acceder y reconocer que no se alcanza el desarrollo social y económico sin el cultural.

En los 70 el ALV se focalizó en la oferta de alternativas formativas dirigidas a la población en edad adulta, formación que se popularizó en el momento (Eurydice, 2018). Durante los 90 ya empezó a hablarse de ALV, el cual tenía un enfoque más amplio que la mera educación de adultos. Con ella se reforzó, por un lado, la importancia de preparar a los estudiantes para aprender por sí mismos y adaptarse a las continuas y cambiantes demandas propias de la sociedad del conocimiento y, por otro lado, también se promovió la educación a distancia mediante la creación en 1992 del Centro para la Innovación y Desarrollo de la Educación a Distancia (CIDEAD). La finalidad de esta institución residía en facilitar el acceso a la educación para los adultos y estudiantes en edad escolar que, debido a circunstancias personales, sociales, geográficas o de otra índole, no les sea posible estudiar de forma presencial en la educación general. 
En 2001 se estableció el Sistema Nacional de Cualificaciones y Formación Profesional (SNCFP) el cual albergaba al Catálogo Nacional de Cualificaciones (CNCP), un instrumento que organiza las calificaciones vocacionales identificadas en el sistema productivo y que permite reconocer y acreditar competencias profesionales adquiridas tanto mediante procesos formativos como mediante la experiencia laboral (Eurydice, 2018). En 2007, se crea el subsistema de formación profesional para el empleo, el cual comprende iniciativas enfocadas a la formación de la población activa con el objetivo de promover una formación que responda a las necesidades y demandas de una economía basada en el conocimiento. En los últimos años, se impulsó el Plan Estratégico de aprendizaje a lo largo de la vida (Ministerio de Educación, Cultura y Deporte, 2014) que presenta las medidas necesarias para cumplir con el objetivo de la Comisión Europea de aumentar el aprendizaje de adultos en el período $2010-2020$.

\section{DISCUSIÓN Y CONCLUSIONES}

Observamos cómo tanto en España como en Alemania, hasta tiempos recientes no se concibe el ALV como tal, sino que primeramente se alude a la una forma de educación dedicada a las personas en edad adulta que no hubieran sido alfabetizadas durante la niñez o que, por circunstancias sociales tuvieran que estudiar en ese momento, para lograr su inclusión social y laboral. Hablamos de unos contextos donde a inicios del siglo XIX reinaba la inestabilidad política, el analfabetismo de gran parte de la población y la necesidad de mano de obra cualificada para la inserción profesional en la Revolución Industrial. Es entonces cuando comienza a hablarse de educación de adultos, educación permanente, educación continua, etc. Digamos que es este proceso de cambio el motor del surgimiento de nuevas estrategias educativas y formativas que den respuesta a las demandas de la sociedad. Llama la atención que mientras la problemática del analfabetismo era común en España y en Alemania en el S. XIX, en el S. XX esto cambia considerablemente. Constatamos cómo en Alemania, durante el periodo del nacional socialismo, parece que las medidas formativas surgidas y propuestas durante la República de Weimar quedaron en gran medida suprimidas, mientras que en España, durante el franquismo, se lleva a cabo una eficaz campaña alfabetizadora en la que además incluyen la estrategia de Educación Permanente. Una vez finalizada la II Guerra Mundial, comprobamos como ya estas formas educativas van cobrando forma en Alemania y en España hacia lo que posteriormente se conocería como ALV, inevitablemente influenciado por la tradición anglosajona y su Lifelong Learning, hasta nuestros días donde cada vez es mayor la oferta formativa en diversos ámbitos y niveles educativos. EI ALV es, en definitiva, un paradigma del cambio y el control social, funciones primordiales de la educación que permite la inclusión social y laboral de colectivos que, de no existir estas medidas, habrían sufrido un considerable riesgo de exclusión.

\section{REFERENCIAS BIBLIOGRÁFICAS}

DfEE. (1998). Recent Thinking in Lifelong Learning. Recuperado de http://dera.ioe.ac.uk/4474/1/RR80.pdf.

Etcheverría Arroyo, F. D. (2007). Educación de adultos, subjetividad y transformaciones sociales: Impacto social, humano y económico de la educación de personas adultas. (Tesis inédita de doctorado). Universidad Complutense, Madrid.

Eurydice. (2018). Germany. Adult Education and Training. Recuperado de https://eacea.ec.europa.eu/national-policies/eurydice/content/adult-education-and-training- 31_en\#AdultEducationTraining.

Eurydice. (2018). Spain. Lifelong Learning Strategy. Recuperado de https://eacea.ec.europa.eu/national-policies/eurydice/ content/lifelong-learning-strategy-79_it Feidel-Mertz, H. (1975). Erwachsenenbildung seit 1945: Ausgangsbedingungen und Entwicklungstendenzen in der Bundesrepublik. Köln: Kiepenheuer und Witsch.

Field, J. (2000). Lifelong Learning and the new Educational Order. Stoke on Trent, Trentham Books.

García Garrido, J. L. (1996). Fundamentos de la Educación Comparada. Madrid: Dykinson.

García Ruíz, M. J. (2005). Aprendizaje a lo largo de la vida. En García Garrido, J. L. y García Ruíz, M. J. Temas Candentes de la Educación en el siglo XXI (pp. 43-82). Madrid: Ediciones Académicas. 
Gómez de Castro, F. (1994). La educación de las personas adultas a lo largo de la historia. Perspectiva nacional e internacional. En Sanz Fernández, F. (Dir.) La formación en educación de personas adultas (pp. 157-200). Madrid: UNED.

Ministerio de Educación, Cultura y Deporte. (2014). Plan Estratégico de Aprendizaje a lo largo de la Vida. Recuperado de https://www.mecd.gob.es/dms/mecd/educacion-mecd/areas-educacion/sistema-educativo/estudios-sistemas-educativos/espanol/especificos/estrategia-competencias-ocde/documentacion/Plan-estrat-gico-de-aprendizaje-a-lolargo-de-la-vida.pdf OECD. (1996). Making Lifelong Learning a Reality for All. Paris: OECD.

Spranger, E. (1960). Wilhelm von Humboldt und die Reform des Bildungswesens . Tübingen: Max Niemeyer Verlag.

Torres, R. M. (2003). Aprendizaje a lo largo de toda la vida: Un nuevo momento y una nueva oportunidad para el aprendizaje y la educación básica de adultos en el Sur. ASDI (Estocolmo), IIZ-DVV (Bonn) y IIEP-UNESCO (Paris).

Über uns. (2018). En Volkhochschule.de. Recuperado de https://www.volkshochschule.de/ueber- uns.html

UNESCO. (1995). La convección sobre los derechos del niño. París: UNESCO. 